\title{
The Ricci Operator and Shape Operator of Real Hypersurfaces in a Non-Flat 2-Dimensional Complex Space Form
}

\author{
Dong Ho Lim ${ }^{1}$, Woon Ha Sohn ${ }^{2}$, Hyunjung Song ${ }^{1}$ \\ ${ }^{1}$ Department of Mathematics, Hankuk University of Foreign Studies, Seoul, Republic of Korea \\ ${ }^{2}$ Department of Mathematics, Yeungnam University, Kyongbuk, Republic of Korea \\ Email: dhlnys@hufs.ac.kr, mathsohn@ynu.ac.kr,hsong@hufs.ac.kr
}

Received November 8, 2012; revised December 15, 2012; accepted January 2, 2013

\begin{abstract}
In this paper, we study a real hypersurface $M$ in a non-at 2-dimensional complex space form $M_{2}(c)$ with $\eta$-parallel Ricci and shape operators. The characterizations of these real hypersurfaces are obtained.
\end{abstract}

Keywords: Real Hypersurface; $\eta$-Parallel Shape Operator; $\eta$-Parallel Ricci Operator; Hopf Hypersurface; Ruled Real Hypersurfaces

\section{Introduction}

A complex $n$-dimensional Kaeherian manifold of constant holomorphic sectional curvature $c$ is called a complex space form, which is denoted by $M_{n}(c)$. As is well-known, a complete and simply connected complex space form is complex analytically isometric to a complex projective space $P_{n}(\mathbb{C})$, a complex Euclidean space $\mathbb{C}^{n}$ or a complex hyperbolic space $H_{n}(\mathbb{C})$, according to $c>0, c=0$ or $c<0$.

In this paper we consider a real hypersurface $M$ in a complex space form $M_{2}(c), c \neq 0$. Then $M$ has an almost contact metric structure $(\phi, g, \xi, \eta)$ induced from the Kaehler metric and complex structure $J$ on $M_{n}(c)$. The structure vector field $\xi$ is said to be principal if $A \xi=\alpha \xi$ is satisfied, where $A$ is the shape operator of $M$ and $\alpha=\eta(A \xi)$. In this case, it is known that $\alpha$ is locally constant ([1]) and that $M$ is called a Hopf hypersurface.

Typical examples of Hopf hypersurfaces in $P_{n}(\mathbb{C})$ are homogeneous ones, R. Takagi [2] and M. Kimura [3] completely classified such hypersurfaces as six model spaces which are said to be $A_{1}, A_{2}, B, C, D$ and $E$. On the other hand, real hypersurfaces in $H_{n}(\mathbb{C})$ have been investigated by J. Berndt [4], S. Montiel and A. Romero [5] and so on. J. Berndt [4] classified all homogeneous Hopf hyersurfaces in $H_{n}(\mathbb{C})$ as four model spaces which are said to be $A_{0}, A_{1}, A_{2}$ and $B$. Further, Hopf hypersurfaces with constant principal curvatures in a complex space form have been completely classified as follows:
Theorem 1.1. ([2]) Let $M$ be a homogeneous real hypersurface of $P_{n}(\mathbb{C})$. Then $M$ is tube of radius $r$ over one of the following Kaeherian submanifolds:

$\left(\mathrm{A}_{1}\right)$ a hyperplane $P_{n-1}(\mathbb{C})$, where $0<r<\frac{\pi}{\sqrt{c}} ;$

$\left(\mathrm{A}_{2}\right)$ a totally geodesic $P_{k}(\mathbb{C})(1 \leq k \leq n-2)$, where $0<r<\frac{\pi}{\sqrt{c}}$

(B) a complex quadric $\mathbb{Q}_{n-1}$, where $0<r<\frac{\pi}{2 \sqrt{c}}$;

(C) $P_{1}(C) \times P_{\frac{n-1}{2}}(C)$, where $0<r<\frac{\pi}{2 \sqrt{c}}$ and $n \geq 5$ is odd;

(D) a complex Grassmann $G_{2.5} C$, where

$0<r<\frac{\pi}{2 \sqrt{c}}$ and $n=9$;

(E) a Hermitian symmetric space $S O(10) / U(5)$, where $0<r<\frac{\pi}{2 \sqrt{c}}$ and $n=15$.

Theorem 1.2. ([4]) Let $M$ be a real hypersurface in $H_{n}(\mathbb{C})$. Then $M$ has constant principal curvatures and $\xi$ is principal if and only if $M$ is locally congruent to one of the followings:

$\left(\mathrm{A}_{0}\right)$ a self-tube, that is, a horosphere;

$\left(A_{1}\right)$ a geodesic hypersphere;

$\left(\mathrm{A}_{2}\right)$ a tube over a totally geodesic $H_{k}(\mathbb{C})(1 \leq k \leq n-1)$; 
(B) a tube over a totally real hyperbolic space $H_{n}(\mathbb{R})$.

A real hypersurface of type $A_{1}$ or $A_{2}$ in $P_{n}(\mathbb{C})$ or type $A_{0}, A_{1}$ or $A_{2}$ in $H_{n}(\mathbb{C})$, then $M$ is said to be of type $A$ for simplicity. As a typical characterization of real hypersurfaces of type $A$, in a complex space form $M_{n}(c)$ was given under the condition

$$
g((A \phi-A \phi) X, Y)=0 \text {, }
$$

for any tangent vector fields $X$ and $Y$ on $M$ by $\mathrm{M}$. Okumura [5] for $c>0$ and S. Montiel and A. Romero [6] for $c<0$. Namely

Theorem 1.3. ([5,6]) Let $M$ be a real hypersurface in $M_{n}(c), c \neq 0, n \geq 2$. It satisfies (1.1) on $M$ if and only if $M$ is locally congruent to one of the model spaces of type $A$.

The holomorphic distribution $T_{0}$ of a real hypersurface $M$ in $M_{n}(c)$ is defined by

$$
T_{0}(p)=\left\{X \in T_{p}(M) \mid g(X, \xi)_{p}=0\right\} .
$$

The following theorem characterizes ruled real hypersurfaces in $M_{n}(c)$.

Theorem 1.4. ([7]) Let $M$ be a real hypersurface in $M_{n}(c), c \neq 0, n \geq 2$. Then $M$ is a ruled real hypersurfaces if and only if $\phi A \phi=0$, or equivalently $g(A X, Y)=0$, for any $X, Y \in T_{0}$.

A $(1,1)$ type tensor field $T$ of $M$ is said to be $\eta$-parallel if

$$
g\left(\left(\nabla_{X} T\right) Y, Z\right)=0
$$

for any vector fields $X, Y$ and $Z$ in $T_{0}$. Real hypersurfaces with $\eta$-parallel shape operator or Ricci operator have been studied by many authors (see [13]). Nevertheless, the classification of real hypersurfaces with $\eta$-parallel shape operator or Ricci operator in $M_{n}(c)$ remains open up to this point. Recently, S.H. Kon and T.H. Loo ([9]) investigated the conditions $\eta$-parallel shape operator.

Theorem 1.5. ([9]) Let $M$ be a real hypersurface of $M_{n}(c), c \neq 0, n \geq 3$. Then the shape operator $A$ is $\eta$-parallel if and only if $M$ is locally congruent to a ruled real hypersurface, or a real hypersurface of type $A$ or $B$.

Also, M. Kimura and S. Maeda ([10]) and Y.J. Suh ([11]) investigated the conditions $\eta$-parallel Ricci operator.

Theorem 1.6. ([10,11]) Let $M$ be a real hypersurface in a complex space form $M_{n}(c), c \neq 0$. Then the Ricci operator of $M$ is $\eta$-parallel and the structure vector field $\xi$ is princial if and only if $M$ is locally congruent to one of the model spaces of type $A$ or type $B$.

As for the structure tensor field $\phi$, shape operator $A$ and $\eta$-parallel, I.-B. Kim, K. H. Kim and one of the present authors ([12]) have proved the following.

Theorem 1.7. ([12]) Let $M$ be a real hypersurface in a complex space form $M_{n}(c), c \neq 0, n \geq 3$. If $M$ has the cyclic $\eta$-parallel shape operator (resp. Ricci operator) and satisfies

$$
g((A \phi-\phi A) X, Y)=0
$$

for any $X$ and $Y$ in $T_{0}$, then $M$ is locally congruent to either a real hypersurface of type $A$ or a ruled real hypersurface (resp. $M$ is locally congruent to a real hypersurface of type $A$ ).

The purpose of this paper is to give some characterizations of real hypersurface satisfying (1.4) and having the $\eta$-parallel shape operator or Ricci operator in $M_{2}(c)$. We shall prove the following.

Theorem 1.8. Let $M$ be a real hypersurface in $a$ complex space form $M_{2}(c), c \neq 0$. If $M$ has the $\eta$ parallel shape operator and satisfies (1.4), then $M$ is locally congruent a ruled real hypersurface.

Theorem 1.9. Let $M$ be a real hypersurface in $a$ complex space form $M_{2}(c), c \neq 0$. If $M$ has the $\eta$ parallel Ricci operator and satisfies (1.4), then $M$ is locally congruent to a real hypersurface of type $A$.

All manifolds in the present paper are assumed to be connected and of class $C^{\infty}$ and the real hypersurfaces are supposed to be orientable.

\section{Preliminaries}

Let $M$ be a real hypersurface immersed in a complex space form $M_{2}(c)$, and $N$ be a unit normal vector field of $M$. By $\tilde{\nabla}$ we denote the Levi-Civita connection with respect to the Fubini-Study metric tensor $\tilde{g}$ of $M_{2}(c)$. Then the Gauss and Weingarten formulas are given respectively by

$$
\tilde{\nabla}_{X} Y=\nabla_{X} Y+g(A X, Y) N, \tilde{\nabla}_{X} N=-A X
$$

for any vector fields $X$ and $Y$ tangent to $M$, where $g$ denotes the Riemannian metric tensor of $M$ induced from $\tilde{g}$, and $A$ is the shape operator of $M$ in $M_{2}(c)$. For any vector field $X$ on $M$ we put

$$
J X=\phi X+\eta(X) N, J N=-\xi,
$$

where $J$ is the almost complex structure of $M_{2}(c)$. Then we see that $M$ induces an almost contact metric structure $(\phi, g, \xi, \eta)$, that is,

$$
\begin{aligned}
& \phi^{2} X=-X+\eta(X) \xi, \phi \xi=0, \eta(\xi)=1, \\
& g(\phi X, \phi Y)=g(X, Y)-\eta(X) \eta(Y), \eta(X)=g(X, \xi)
\end{aligned}
$$

for any vector fields $X$ and $Y$ on $M$. Since the almost complex structure $J$ is parallel, we can verify from the Gauss and Weingarten formulas the followings: 


$$
\begin{gathered}
\nabla_{X} \xi=\phi A X, \\
\left(\nabla_{X} \phi\right) Y=\eta(Y) A X-g(A X, Y) \xi .
\end{gathered}
$$

Since the ambient manifold is of constant holomorphic sectional curvature $c$, we have the following Gauss and Codazzi equations respectively:

$$
\begin{aligned}
R(X, Y) Z \\
=\frac{c}{4}\{g(Y, Z) X-g(X, Z) Y+g(\phi Y, Z) \phi X \\
\quad-g(\phi X, Z) \phi Y-2 g(\phi X, Y) \phi Z\} \\
\quad+g(A Y, Z) A X-g(A X, Z) A Y, \\
\\
\quad\left(\nabla_{X} A\right) Y-\left(\nabla_{Y} A\right) X \\
=\frac{c}{4}\{\eta(X) \phi Y-\eta(Y) \phi X-2 g(\phi X, Y) \xi\}
\end{aligned}
$$

for any vector fields $X, Y$ and $Z$ on $M$, where $R$ denotes the Riemannian curvature tensor of $M$. From (1.3), the Ricci operator $S$ of $M$ is expressed by

$$
S X=\frac{c}{4}\{(2 n+1) X-3 \eta(X) \xi\}+m A X-A^{2} X,
$$

where $m=\operatorname{trace} A$ is the mean curvature of $M$, and the covariant derivative of (2.5) is given by

$$
\begin{aligned}
& \left(\nabla_{X} S\right) Y \\
= & -\frac{3 c}{4}\{g(\phi A X, Y) \xi+\eta(Y) \phi A X\}+(X m) A Y \\
& +m\left(\nabla_{X} A\right) Y-\left(\nabla_{X} A\right) A Y-A\left(\nabla_{X} A\right) Y .
\end{aligned}
$$

Let $\mathrm{U}$ be a unit vector field on $M$ with the same direction of the vector field $-\phi \nabla_{\xi} \xi$, and let $\mu$ be the length of the vector field $-\phi \nabla_{\xi} \xi$ if it does not vanish, and zero (constant function) if it vanishes. Then it is easily seen from (1.1) that

$$
A \xi=\alpha \xi+\beta U,
$$

where $\alpha=\eta(A \xi)$. We notice here that $U$ is orthogonal to $\xi$. We put

$$
\Omega=\{p \in M \mid \beta(p) \neq 0\} .
$$

Then $\Omega$ is an open subset of $M$.

\section{Some Lemmas}

In this section, we assume that $\Omega$ is not empty, then there are sclar fields $\gamma, \varepsilon$ and $\delta$ and a unit vector field $U$ and $\phi U$ orthogonal to $\xi$ such that

$$
A U=\beta \xi+\gamma U+\varepsilon \phi U, A \phi U=\varepsilon U+\delta \phi U
$$

and

$$
m=\operatorname{trace} A=\alpha+\gamma+\delta
$$

in $M_{2}(c)$. We shall prove the following Lemmas.
Lemma 3.1. Let $M$ be a real hypersurface in a complex space form $M_{2}(c), c \neq 0$. If $M$ satisfies (1.4), then we have $A U=\beta \xi+\gamma U, A \phi U=\delta \phi U$ and $\delta=\gamma$.

Proof. If we put $X=Y=U$, or $X=U$ and $Y=\phi U$ into (1.4) and make use of (3.1), then we have

$$
\varepsilon=0 \text { and } \delta=\gamma .
$$

Therefore, it follows that $A U$ is expressed in terms of $\xi$ and $U$ only and $A \phi U$ given by $\phi U$. $\square$

It follows from (2.6), (2.8) and Lemma 3.1 that

$$
\begin{aligned}
& S \xi=\left(\frac{c}{2}+2 \alpha \gamma-\beta^{2}\right) \xi+\beta \gamma U, \\
& S U=\beta \gamma \xi+\left(\frac{5 c}{4}+\alpha \gamma-\beta^{2}+\gamma^{2}\right) U, \\
& S \phi U=\left(\frac{5 c}{4}+\alpha \gamma+\gamma^{2}\right) \phi U .
\end{aligned}
$$

Lemma 3.2. Under the assumptions of Lemma 3.1. If $M$ has the $\eta$-parallel Ricci operator $S$ then we have $U \beta=0$ and $(\phi U) \beta=-\gamma^{2}$.

Proof. Differentiating the second of (3.4) covariantly along vector field $X$ in $T_{0}$, we obtain

$$
\begin{aligned}
& \left(\nabla_{X} S\right) U \\
= & \left\{\left(\frac{5 c}{4}+\alpha \gamma-\beta^{2}+\gamma^{2}\right) I-S\right\} \nabla_{X} U+\beta \gamma \phi A X \\
& +X(\beta \gamma) \xi+X\left(\frac{5 c}{4}+\alpha \gamma-\beta^{2}+\gamma^{2}\right) U .
\end{aligned}
$$

Taking inner product of (3.5) with $U$ and $\phi U$ and making use of (3.5) and Lemma 3.1, we have

$$
2 \beta \gamma^{2} g(\phi U, X)=X\left(\frac{5 c}{4}+\alpha \gamma-\beta^{2}+\gamma^{2}\right)
$$

and

$$
\beta g\left(\nabla_{X} U, \phi U\right)=\gamma^{2} g(U, X) .
$$

If we put $X=U$ and $Y=\phi U$ into (3.6) then we have

$$
(\alpha+2 \gamma) U \gamma+\gamma U \alpha-2 \beta U \beta=0
$$

and

$$
2 \beta \gamma^{2}=(\alpha+2 \gamma)(\phi U) \gamma+\gamma(\phi U) \alpha-2 \beta(\phi U) \beta .
$$

Putting $X=U$ and $Y=\phi U$ into (3.7), then we obtain

$$
\beta g\left(\nabla_{U} U, \phi U\right)=\gamma^{2} \text { and } \beta g\left(\nabla_{\phi U} U, \phi U\right)=0 .
$$

If we differentiate the third of (3.4) covariantly along vector field $X$ in $T_{0}$, we obtain 


$$
\begin{aligned}
\left(\nabla_{X} S\right) \phi U & =\left\{\left(\frac{5 c}{4}+\alpha \gamma+\gamma^{2}\right) I-S\right\} \nabla_{X} \phi U \\
& +\left\{X\left(\frac{5 c}{4}+\alpha \gamma+\gamma^{2}\right)\right\} \phi U .
\end{aligned}
$$

If we take inner product of $\phi U$ and using (3.4), then we have

$$
X\left(\frac{5 c}{4}+\alpha \gamma+\gamma^{2}\right) \phi U=0
$$

Substituting $X=U$ and $\phi U$ into (3.12), we obtain

$$
\begin{aligned}
& (\alpha+2 \gamma) U \gamma+\gamma U \alpha=0 \text { and } \\
& (\alpha+2 \gamma)(\phi U) \gamma+\gamma(\phi U) \alpha=0 .
\end{aligned}
$$

By comparing (3.8) and (3.9) with (3.13), we have $U \beta=0$ and $(\phi U) \beta=-\gamma^{2}$.

Lemma 3.3. Under the assumptions of Lemma 3.2, we have $\nabla_{X} U=\gamma g(\phi U, X) \xi+\frac{\gamma^{2}}{\beta} g(U, X) \phi U$.

Proof. Since we have $A \phi U=\gamma \phi U$ and using (3.7), we get

$$
\begin{aligned}
& a(X)=g\left(\nabla_{X} U, \xi\right)=\gamma(\phi U, X) \text { and } \\
& c(X)=g\left(\nabla_{X} U, \phi U\right)=\frac{\gamma^{2}}{\beta} g(U, X) .
\end{aligned}
$$

Thus, it follows from (3.14) that

$\nabla_{X} U=\gamma g(\phi U, X) \xi+\frac{\gamma^{2}}{\beta} g(U, X) \phi U$.

Lemma 3.4. Under the assumptions of Lemma 3.2, we have $\xi \alpha=\xi \beta=\xi \gamma=0$ and $U \alpha=U \gamma=0$.

Proof. Differentiating the smooth function $\alpha=g(A \xi, \xi)$ along any vector field $X$ on $\Omega$ and using (2.2) and (2.5) and Lemma 3.1, we have

$$
X \alpha=g\left(\left(\nabla_{\xi} A\right) \xi-2 \beta \gamma \phi U, X\right) .
$$

Since we have $\left(\nabla_{\xi} A\right) \xi=\nabla_{\xi}(\alpha \xi+\beta U)-A \nabla_{\xi} \xi$, we see from this equation above that the gradient vector field $\nabla \alpha$ of $\alpha$ is given by

$$
\nabla \alpha=\beta \nabla_{\xi} U+(\xi \alpha) \xi+(\xi \beta) U+(\alpha \beta-3 \beta \gamma) \phi U .
$$

If we put $X=\xi$ into Lemma 3.3, then we have

$$
\nabla_{\xi} U=0 \text {. }
$$

Thus, the above equation is reduced to

$$
\nabla \alpha=(\xi \alpha) \xi+(\xi \beta) U+(\alpha \beta-3 \beta \gamma) \phi U .
$$

Taking inner product of this equation with $U$ and $\phi U$ respectively, we obtain

$$
U \alpha=\xi \beta \text { and }(\phi U) \alpha=\alpha \beta-3 \beta \gamma .
$$

If we differentiate the smooth function $\beta=g(A U, \xi)$ along any vector field $X$ on $M$ and using (2.2), (2.5) and (2.8) and Lemma 3.2, we have

$$
\nabla \beta=\beta \nabla_{U} U+(U \alpha) \xi+(U \beta) U+\left(\frac{c}{2}+2\left(\alpha \gamma-\gamma^{2}\right)\right) \phi U
$$

Putting $X=U$ into Lemma 3.3, then we have

$$
\nabla_{U} U=\frac{\gamma^{2}}{\beta} \phi U
$$

If we substitute (3.20) into (3.19), then we obtain

$$
\nabla \beta=(U \alpha) \xi+(U \beta) U+\left(\frac{c}{2}+2 \alpha \gamma-\gamma^{2}\right) \phi U .
$$

If we take inner product of this equation with $\phi U$ and using $(\phi U) \beta=-\gamma^{2}$ in Lemma 3.2, then we have

$$
\alpha \gamma+\frac{c}{4}=0
$$

As a similar argument as the above, we can verify that the gradient vector fields of the smooth function $\gamma=g(A U, U)=g(A \phi U, \phi U)$ is given respectively by

$$
\nabla \gamma=-(A-\gamma I) \nabla_{U} U+(U \beta) \xi+(U \gamma) U+3 \beta \gamma \phi U
$$

and

$$
\nabla \gamma=((\phi U) \gamma) \phi U
$$

by virtue of (2.3) and Lemma 3.2.

If we substitute (3.24) into (3.23) and make use of (3.20) and Lemma 3.1, then we obtain

$$
(U \beta) \xi+(U \gamma) U-((\phi U) \gamma-3 \beta \gamma) \phi U=0 .
$$

If we take inner product of this equation with $U$ and $\phi U$ respectively, then we have

$$
U \gamma=0 \text { and }(\phi U) \gamma=3 \beta \gamma .
$$

If we substitute (3.26) into (3.14) and take account of (3.21), then we have $U \alpha=0$. Also, if we differentiate (3.21) along any vector field $\xi$, then we have

$$
\alpha \xi \gamma+\gamma \xi \alpha=0 \text {. }
$$

Taking inner product of (3.23) with $\xi$ and using (3.18), we get $\xi \gamma=U \beta$. Since $U \alpha=0$, we see from (3.27) and the first of (3.18) that $\xi \gamma=0, \xi \alpha=0$ and $\xi \beta=0$.

\section{Proofs of Theorems}

Proof Theorem 1.8. If (1.4) is given in $M$, then we see that Lemma 3.1 holds on $M$. If we differentiate (1.3) along any vector field $X$ in $T_{0}$ and using (2.3) and (2.8), then we have 


$$
\begin{aligned}
& g\left((A \phi-\phi A) Z, \nabla_{X} Y\right)+g\left((A \phi-\phi A) Y, \nabla_{X} Z\right) \\
& =\beta(g(U, Z) g(A X, Y)+g(U, Y) g(A X, Z))
\end{aligned}
$$

for any vector fields $X, Y$ and $Z$ on $T_{0}$. Putting $X=Y=Z=U$ into (4.1) and using Lemma 3.1 and 3.3, then we have

$$
\beta \gamma=0 .
$$

Since $\Omega$ is not empty, we have $\gamma=0$ hold on $\Omega$. It follows from (2.8) and Lemma 3.1 that

$$
A \xi=\alpha \xi+\beta U, A U=\beta \xi \text { and } A \phi U=0 .
$$

Thus $M$ is locally congruent to ruled real hypersurface (see [7]). $\square$

Proof Theorem 1.9. Assume that the open set $\Omega=\{p \in M \mid \beta(p) \neq 0\}$ is not empty. Then we consider from Lemma 3.2 and 3.3 that $(\phi U) \beta=-\gamma^{2}$ and $c(U)=\frac{\gamma^{2}}{\beta}$. If we differentiate the smooth function $\beta=g(A \xi, U)$ along vector field $X$ on $M$ and (2.2), (2.5) and (2.8), we have

$$
X \beta=g\left(\left(\nabla_{\xi} A\right) U+\left(\frac{c}{4 \alpha}+\alpha \gamma-\gamma^{2}\right) \phi U, X\right) .
$$

Since we have $\left(\nabla_{\xi} A\right) U=\nabla_{\xi}(\beta \xi+\gamma U)-A \nabla_{\xi} U$, we see from this equation above that gradient vector field $\nabla \beta$ of $\beta$ is given by

$$
\begin{aligned}
\nabla \beta= & -(A-\gamma I) \nabla_{\xi} U+(\xi \beta) \xi+(\xi \gamma) U \\
& +\left(\beta^{2}+\frac{c}{4}+\alpha \gamma-\gamma^{2}\right) \phi U,
\end{aligned}
$$

where $I$ indicates the identity transformation on $M$. If we substitute (3.16) into (4.4) and using Lemma 3.4, then we obtain

$$
\nabla \beta=\left(\beta^{2}+\frac{c}{4}+\alpha \gamma-\gamma^{2}\right) \phi U .
$$

Since we have $(\phi U) \beta=-\gamma^{2}$, we get

$$
\beta^{2}+\frac{c}{4}+\alpha \gamma=0
$$

By (4.6) and (3.22), we have $\beta=0$ and hence it is a contradiction. Thus the set $\Omega=\{p \in M \mid \beta(p) \neq 0\}$ is empty, and hence $M$ is a Hopf hypersurface. Since $M$ is a Hopf hypersurface, we see from (2.1) and (2.8) that $(A \phi-\phi A) \xi=0$, which together with our assumption (1.4) implies (1.1), that is $A \phi=\phi A$ on $M$. Thus, Theorem 1.9 shows that $M$ is locally congruent to a real hypersurface of type $A$. $\square$

\section{Acknowledgements}

The authors would like to express their sincere gratitude to the refree who gave them valuable suggestions and comments.

\section{REFERENCES}

[1] U.-H. Ki and Y. J. Suh, "On Real Hypersurfaces of a Complex Space Form," Mathematical Journal of Okayama University, Vol. 32, 1990, pp. 207-221.

[2] R. Takagi, "On Homogeneous Real Hypersurfaces in a Complex Projective Space," Osaka Journal of Mathematics, Vol. 10, 1973, pp. 495-506.

[3] M. Kimura, "Real Hypersurfaces and Complex Submanifolds in Complex Projective Space," Transactions of the American Mathematical Society, Vol. 296, 1986, pp. 137 149. doi:10.1090/S0002-9947-1986-0837803-2

[4] J. Berndt, "Real Hypersurfaces with Constant Principal Curvatures in Complex Hyperbolic Space," Journal Für Die Reine und Angewandte Mathematik, Vol. 1989, No. 395, 1989, pp. 132-141.

[5] M. Okumura, "On Some Real Hypersurfaces of a Complex Projective Space," Transactions of the American Mathematical Society, Vol. 212, 1975, pp. 355-364. doi:10.1090/S0002-9947-1975-0377787-X

[6] S. Montiel and A. Romero, "On Some Real Hypersurfaces of a Complex Hyperbolic Space," Geometriae Dedicata, Vol. 20, No. 2, 1986, pp. 245-261. doi:10.1007/BF00164402

[7] S. Maeda and T. Adachi, "Integral Curves of Characteristic Vector Fields of Real Hypersurfaces in Nonflat Complex Space Forms," Geometriae Dedicata, Vol. 123, No. 1, 2006, pp. 65-72. doi:10.1007/s10711-006-9100-1

[8] W. H. Sohn, "Characterizations of Real Hypersurfaces of Complex Space Forms in Terms of Ricci Operators," Bulletin of the Korean Mathematical Society, Vol. 44, No. 2007, pp. 195-202. doi:10.4134/BKMS.2007.44.1.195

[9] S. H. Kon and T. H. Loo, "Real Hypersurfaces of a Complex Space Form with $\eta$-Parallel Shape Operator," Mathematische Zeitschrift, Vol. 269, No. 1-2, 2011, pp. 47-58. doi:10.1007/s00209-010-0715-4

[10] M. Kimura and S. Maeda, "On Real Hypersurfaces of a Complex Projective Space," Mathematische Zeitschrift, Vol. 202, No. 3, 1989, pp. 299-311. doi:10.1007/BF01159962

[11] Y. J. Suh, "On Real Hypersurfaces of a Complex Space Form with $\eta$-Parallel Ricci Tensor," Tsukuba Journal of Mathematics, Vol. 14, 1990, pp. 27-37.

[12] I.-B. Kim, K. H. Kim and W. H. Sohn, "Characterizations of Real Hypersurfaces in a Complex Space Form," Canadian Mathematical Bulletin, Vol. 50, 2007, pp. 97-104. doi:10.4153/CMB-2007-009-5 